Working Paper 92-03

February 1992
División de Economía

Universidad Carlos III de Madrid

Calle Madrid, 126

28903 Getafe (Madrid)

\title{
A SIMPLE METHOD TO IDENTIFY SIGNIFICANT EFFECTS IN UNREPLICATED TWO-LEVEL FACTORIAL DESIGNS
}

\author{
Jesús Juan and Daniel Peña*
}

\begin{abstract}
This article proposes a generalization and improvement on the method of Lenth (1989). The problem is solved by fixing outliers in highly contaminated samples. To do this a scale robust estimator is obtained and its performance is analyzed using computer simulations. The method is extremely simple to use and leads to the same results as the more complex one proposed by Box and Meyer (1986).
\end{abstract}

Key words: factorial fractions, robust estimator.

* Jesús Juan, Laboratorio de Estadística, E.T.S. Ingenieros Industriales, Universidad Politécnica de Madrid. Daniel Peña, Departamento de Estadística y Econometría, Universidad Carlos III de Madrid and Laboratorio de Estadística, Universidad Politécnica de Madrid. 


\section{INTRODUCTION}

In industral applications, it is often necessary to determine which factors among a great number of possibilities can affect a process. As factorial designs require a number of runs that grows exponentially with the number of factors to be analysed, the application of full replicated factorial designs is both costly and unnecessary, and the sequential use of factorial designs is to be recommended, as it allows the identification of more significant effects and considerably reduces the number of runs to be carried out.

In the analysis of this type of design the absence of replications makes it impossible to estimate the residual variance, the latter being necessary to contrast which factors and interactions are active. In full factorial designs, or in high-resolution designs, the higher order interactions can be supposed not active, and the squared mean of their estimates can be used as the residual variance estimator. This procedure, apart from other disadvantages, is not applicable to low-resolution fractions (for example, saturated designs), since there are no interactions whose estimates can be used in the calculation of the scale estimator.

The first acceptable solution for the analysis of unreplicated designs was suggested by Daniel $(1959,1976)$. His method consists of drawing on normal probability paper the estimates of the effects: on the graph, the correspondent estimates to non-active columns (the majority) form an approximately straight line and the significant effects appear at a distance as outliers in a regression line. This procedure has been of great use and, with difference, the most recommended (see for example, Box, Hunter and Hunter, 1978). The main inconvenience of the graph is its subjectivity, and frequently only experienced analysts can judge if an apparent deviation from the linearity is significant or not.

Box and Meyer (1986) approached the problem from a more formal point of view, based on the Pareto principle, "only a reduced number of effects are significant." Accepting the hypothesis that the estimated effects come from 
a normal contaminated distribution, $(1-\alpha) \mathrm{N}\left(0, \sigma^{2}\right)+\alpha \mathrm{N}\left(0, k^{2} \sigma^{2}\right)$ where $\alpha$ and $k$ are supposedly known, the probability that an effect is active can be calculated by Bayes's theorem. The necessary calculations involved in the application of this method are complex and it is essential to make use of specialized programs in order to carry them out.

Peña (1987) suggested judging what effects are significant, by using as a reference a robust estimate of the scale parameter. The procedure is extremely simple and provides the same results as the far more complex procedure of Box and Meyer's Bayesian plot. In Peña and Juan (1989) this procedure is presented, proposing as scale estimator the median of the absolute value of the estimates. This article studies the properties of this method in depth, justifying each one of the decisions taken. An analysis based on simulations on a computer proves that the procedure is very powerful and this together with its simplicity makes it a useful complement to the existing methods.

Benski (1989) applies a normality test (Olsson 1979, 1981) in order to identify the significant effects, combined with a test of outliers that uses the fourth-spread as a scale robust estimator. The author himself recognizes, "in fact, the fourth-spread outlier test has shown to be a useful tool on its own since it can also identify the same significant effects, when applied to the same data set as the normality test." In point 3 the advantages of taking the median of the absolute values as a scale estimator are determined as opposed to the fourth-spread.

Wang and Lawson (1988) using the Bayesian approach, obtain an estimate of the residual variance. To apply this it is essential to use a sophisticated computer program, and this requirement has the same problem as Box and Meyer's method.

Finally, Lenth (1989) has suggested a related method to the one presented in this article. Both procedures will be compared in section 5 .

In this article an effect refers in general to any column of the design 
matrix. In the case of fractions of factorial designs, each column or contrast will consist of a linear combination of effects defined by the aliases.

\section{FUNDAMENTALS}

If we accept the hypothesis that only a reduced number of effects are active (Pareto principle), their identification in unreplicated factorial designs is reduced to the problem of identifying the outliers of a sample. The problem from this new point of view is the following: consider a factorial design, $2^{h-p}$, being $N=2^{h-p}$ the number of runs. Let $\left(\hat{\theta}_{1}, \hat{\theta}_{2}, \ldots, \hat{\theta}_{n}\right)$ be the estimates of the effects except the mean, $n=N-1$. A generally accepted hypothesis is that these estimates come from normal distributions with possibly different means, but with the same unknown variance. That is, if the effect $i$ is not active, $\hat{\theta}_{i}$ comes from a $\mathrm{N}\left(0, \sigma^{2}\right)$ distribution, and if it is active it comes from $N\left(\theta_{i}, \sigma^{2}\right)$, where $\theta_{i}$ is a nonzero real number. If we suppose that a priori the set of parameters $\theta_{i}$ corresponding to active effects follows a $\mathrm{N}\left(0, \sigma_{\theta}^{2}\right)$ with $\sigma_{\theta} \gg \sigma$, the predictive distribution of $\hat{\theta}_{i}$, if $i$ is active, is $\mathrm{N}\left(0, \sigma^{2}+\sigma_{\theta}^{2}\right)$.

Let $\alpha$ be the probability that an effect is active, then $\left(\hat{\theta}_{1}, \hat{\theta}_{2}, \ldots, \hat{\theta}_{n}\right)$ are independent and identically distributed values of a contaminated normal distribution (Box and Meyer, 1986)

$$
(1-\alpha) \mathrm{N}\left(0, \sigma^{2}\right)+\alpha \mathrm{N}\left(0, \mathrm{k}^{2} \sigma^{2}\right)
$$

where

$$
k^{2}=\left(\sigma^{2}+\sigma_{\theta}^{2}\right) / \sigma^{2}
$$

The problem, bearing in mind the previous contaminated model, is reduced to estimating $\sigma$ avoiding the influence of contaminating "observations" (active effects). One solution is to use robust estimators for $\sigma$ with a high break-down point (see Hampel et al, 1986) which are efficient with high levels of contamination (Box and Meyer estimated for a set of published examples that the contamination level $\alpha$ is in the neighborhood of 0.1 to 0.3 ). 


\section{ROBUST SCALE ESTIMATORS}

Three robust scale estimators of frequent use (Iglewicz, 1983) for $\left(\hat{\theta}_{1}, \hat{\theta}_{2}, \ldots, \hat{\theta}_{n}\right)$ are:

1. The median of the absolute deviation to the sample median

$$
M A D=\operatorname{median}\left\{\left|\hat{\theta}_{i}-M\right|\right\},
$$

where $M=\operatorname{median}\left\{\hat{\theta}_{i}\right\}$

2. The median of the absolute value of the observation

$$
M A D_{0}=\operatorname{median}\left\{\left|\hat{\theta}_{i}\right|\right\}
$$

3. The fourth-spread; difference between the third and first quartile.

$$
d_{F}=F_{U}-F_{L}
$$

If we wish to estimate the standard deviation $\sigma$, we should standardize these estimators in such a way that they are consistent in the case of the normal distribution. To do this, we must divide $M A D$ and $M A D_{0}$ by $\Phi^{-1}(3 / 4)=0.6745$ and $d_{F}$ by $2 \Phi^{-1}(3 / 4)=1.349$, where $\Phi$ is the distribution function of the standard normal.

The comparison of performance for these three estimators in the case of the proposed contaminated model has been carried out by the Monte Carlo method, with designs of 16 and 32 runs, values of $\alpha=0$ to $\alpha=0.30$ and values of $k=5,10$ and 15 . In each case 1000 simulations were performed.

The results for different combinations of the sample size and $k$ are similar, consequently only the results for the case $k=10$ and $n=15$ will be commented on. Figure 1a shows the graphs of the average bias estimated as a function of the value $\alpha$. The three estimators tend to overestimate $\sigma$, the bias growing as $\alpha$ grows. We may conclude from these simulations that for high levels of contamination the three robust estimates have considerably high bias which make their use not very recommendable, especially the 
fourth-spread which with $\alpha=0.3$ produces an average positive bias of $70 \%$, due without doubt to its smaller break-down point (0.25).

Given the differences of observed bias it would seem advisable to compare these three estimates from the mean squared error (MSE) point of view. Figure $1 \mathrm{~b}$ represents the graph of the MSE obtained in the simulations for these three estimates as a function of $\alpha$. A log-scale is used for the MSE to enhance the resolution of the plot. The similarity between $M A D$ and $M A D_{0}$ can be appreciated: the smaller bias of $M A D$ is compensated by the smaller variance of $M A D_{0}$, and confirms the bad behaviour of $d_{F}$, which is not only the most biased but also the least efficient. The first conclusion obtained from the simulations is the difference of performance between $d_{F}$ and the other two estimators: On the other hand, when the percentage of outliers is high, none of the robust estimators studied have good properties.

\section{PROPOSED METHOD}

\subsection{An Iterative Estimator}

In order to confirm if an iterative procedure improves the previous results, a new robust estimate $I M A D_{0}\left(M A D_{0}\right.$ iterative) is defined as follows:

1. Compute $M A D_{0}$ using (2) with the $n$ estimates of the effects,

2. Take those values $\hat{\theta}_{i}$ which satisfy

$$
\left|\hat{\theta}_{i}\right| \leq w M A D_{0}
$$

$w$ being a previously determinated value which, as will be justified in the Appendix, must be such that $w>2$.

3. With those values recalculate $M A D_{0}$. If the new estimate is different from the previous one, repeat step 2, otherwise, the procedure terminates and the last $M A D_{0}$ is the $I M A D_{0}$. 
As in the case of the robust estimators in section 3 , it is necessary to divide the new scale estimator by a correction factor $a_{w}$ in order to obtain a consistent estimator for $\sigma$ in the normal case.

$$
\hat{\sigma}=\frac{I M A D_{0}}{a_{w}}
$$

This correction factor is a function of $w$ and it is obtained for any $w>2$ as solution $t>0$ of the equation (see Appendix)

$$
\Phi(t)=\frac{1}{2} \Phi(w t)+\frac{1}{4}
$$

The solution for the previous equation is obtained recursively in a few iterations. Note that for $w \rightarrow \infty, a_{w}=\Phi^{-1}(3 / 4)$, and for other values of $w$ it will satisfy $a_{w}<\Phi^{-1}(3 / 4)$. In Table I the solution of equation (6) for different values of $w$ is shown.

TABLE I. Solution $t=a_{w}$ of equation (6) for different values of $w$.

\begin{tabular}{|c|c|c|c|c|c|c|c|c|c|}
\hline$w$ & 2.5 & 3 & 3.5 & 4 & 4.5 & 5 & 5.5 & 6 & 6.5 \\
\hline$a_{w}$ & 0.5424 & 0.6285 & 0.6578 & 0.6686 & 0.6725 & 0.6739 & 0.6743 & 0.6744 & 0.6745 \\
\hline
\end{tabular}

The most adequate value of $w$ will be the one which provides an estimator with smallest bias and MSE. To determine this value some simulations have been carried out for different combinations of $n, k$ and $\alpha$ following the contaminated model. For each of the samples the value of $I M A D_{0}$ was calculated for values of $w$ varying from 2.5 to 8 ; this procedure was repeated 1000 times. Figures 2a to $2 \mathrm{~d}$ include graphs for average bias and MSE of the standardized estimator (divided by $a_{w}$ ) for non-contaminated samples 1 $(n=7), 2(n=15), 3(n=31)$, and for contaminated samples $4(n=7$, $\alpha=0.1, k=5), 5(n=15, \alpha=0.2, k=10)$ and $6(n=31, \alpha=0.3$, $k=15$ ). It can be seen how (Fig. 2a) the iterative estimator is biased even for the non-contaminated model and how the bias becames smaller as the sample size increases. The three graphs present a minimum for $w$ in the neighborhood of 3 to 4 . As expected, the bias is larger for the contaminated 
models (Fig. 2b) showing a shift upwards of the graphs if one compares them with those corresponding to the non-contaminated samples, in these graphs one can observe more clearly the existence of a minimum. Figure 2c shows the decreasing MSE curves of the estimator for non-contaminated samples. If contamination is present (Fig. 2d), the MSE increases; a rise and the existence of minumum points can be seen in the graphs. Figures $2 \mathrm{~b}$ and $2 \mathrm{~d}$ show how the value $w$ which minimizes the MSE does not coincide with the one that minimizes the bias. The election of $w$ will ultimately depend on the criterion followed, either minimum bias or minimum MSE, and on the sample size. In the examples shown and in others analysed the optimal $w$ value becames smaller as the sample size increases. On the other hand, any value of $w$ in the interval $(3,4)$ provides acceptable values for the bias and the MSE; the value of $w=3.5$ is recommended for the most frequent designs of 8 to 64 runs and it has been chosen to analyse the examples presented in section 6.

\subsection{Comparison with other estimators}

Figure 1a also shows the graph of the iterative estimator $(w=3.5)$ as a function of $\alpha$. The bias is considerably smaller than in the previous cases and the rate of growth for increasing values of $\alpha$ is fairly moderate. IMAD has an average bias of $7.8 \%$ for $\alpha=0.25$, as opposed to the $33 \%$ bias of $M A D_{0}$. These differences of behaviour remain favourable to the iterative estimate when we compare them in terms of MSE, as shown in Figure 1b. For the maximum value of alpha utilized (0.3), the iterative estimator is about $20 \%$ more efficient than $M A D_{0}$.

The previous procedure for calculating $I M A D_{0}$ at the same time provides the set $I$ of observations which are considered as outliers according to this criterion, $I=\left\{i:\left|\hat{\theta}_{i}\right|>w I M A D_{0}\right\}$. If we eliminate these observations from the sample, the rest are, hypothetically, independent observations, identically distributed from a Normal distribution with mean 0 and unknown 
variance, $\sigma^{2}$. In this case, the most efficient estimate for the variance is the one obtained as the mean square of the observations,

$$
\hat{s}_{R}^{2}=\frac{\sum_{i \notin I} \hat{\theta}_{i}^{2}}{(n-m)},
$$

where $m$ is the number of outliers. It is known that this value coincides with that obtained through the residual variance of the model which only takes into account the estimated active effects. Figure 3 represents the MSE of this estimator $\left(\hat{s}_{R}\right)$ and its comparison with the $I M A D_{0}$ estimator. For levels of contamination lower than $20 \%, \hat{s}_{R}$, as expected, has a lower MSE than the robust estimator. However, this difference is reversed and grows when the level of contamination $\alpha$ is raised above 0.2 . This result, which in principle can seem surprising, has a simple interpretation: when $\alpha$ is large there exists a high probability of observations generated by the normal of greater variance appearing, and not being considered outliers as they appear within the defined limits. These observations are indistinguishable from the "good" observations and inflate $\hat{s}_{R}$, while they have lesser effect on the robust estimate. This leads us to the following conclusion: once the outliers are identified the robust estimator is preferable to $\hat{s}_{R}$, especially when the number of detected outliers is large (above $20 \%$ ).

\subsection{Testing for outliers}

Once we have a reasonably good estimator of the variance, the next step is to define a testing procedure based on this estimator so that we can decide which effect is active. A rule based on the principle of simultaneous testing of $n$ contrast that yields acceptable practical results is considering active those effects satisfying

$$
\left|\hat{\theta}_{i}\right| \geq z_{c} \hat{\sigma}
$$

where, given $\beta, z_{c}$ is obtained by

$$
P\left(|Z| \leq z_{c}\right)=(1-\beta)^{\frac{1}{n}},
$$


where $Z$ is a random variable $\mathrm{N}(0,1)$, (that is $\left.z_{c}=\Phi^{-1}\left(\frac{1+(1-\beta)^{1 / n}}{2}\right)\right)$. In most regular designs of 8,16 and 32 runs, if $\beta=0.05$, then $z_{c}$ is equal to $2.68,2.93$ and 3.15, respectively. Using (5) and defining $w_{c}=z_{c} / a_{w}$, the inequality (7) can be written as

$$
\left|\hat{\theta}_{i}\right| \geq w_{c} I M A D_{0}
$$

and if we use the recommended $w=3.5$, then $w_{c}$ will take the values $4,4.4$ and 4.8 for the designs with 8,16 and 32 runs, respectively.

\section{COMPARISON WITH LENTH'S METHOD}

If the iterative process to calculate $I M A D_{0}$ is stopped in the second iteration, making $w=3.75$, the robust estimate obtained is the same as the one proposed by Lenth (1989). This estimate was named PSE (pseudo standard error) by its author. Both estimates behave similarly when the level of contamination is low, but as $\alpha$ increases, the bias and the MSE of IMAD $D_{0}$ tend to decrease. In Figure $4 \mathrm{a}$ a comparison is made between the biases of the two estimators; $I M A D_{0}$ has a smaller bias than PSE for any level of contamination, but $P S E$ is less variable when $\alpha$ is small, for large values of $\alpha, I M A D_{0}$ has both a smaller bias and less variability (Fig. 4b).

The behaviour of the estimates in the limit case (when $n \rightarrow \infty$ ) can help to compare with the finite size sample. The limiting values of $I M A D_{0}$ will depend on the model parameters $\alpha, k, \sigma$ and the chosen value for $w$. When $\alpha=0$, assuming without loss of generality that $\sigma=1, a_{w}$ is obtained for any $w>2$ as the solution $t>0$ of the equation (6). In the case of a mixture of normals, $\alpha \neq 0, a_{w}$ is obtained in the same way from the solution $t>0$ of (see Appendix)

$$
\begin{aligned}
\Phi(t)= & \frac{1}{2(1-\alpha)}-\frac{\alpha}{1-\alpha} \Phi\left(\frac{t}{k}\right) \\
& +\frac{1}{4(1-\alpha)}\left((1-\alpha)(2 \Phi(w t)-1)+\alpha\left(2 \Phi\left(\frac{w t}{k}\right)-1\right)\right) .
\end{aligned}
$$

For $0 \leq \alpha \leq 0.3$ and $k \geq 5$, it can be shown (Lenth, 1989) that in the 
worst case $(k=5, \alpha=0.3)$ the limiting value of $P S E$ is $15 \%$ greater than $\sigma$, while with the iterative estimator this bound is reduced to $8 \%$.

\section{EXAMPLES}

In order to illustrate the suggested procedure, four examples of factorial designs are analysed. These examples have been analysed by Box and Meyer using the Bayes plot and were presented and solved in Daniel (1976), Taguchi and Wu (1980), Box, Hunter and Hunter (1978), and Davies (1954). Table II presents these four sets of data and Table III the estimated effects.

We will illustrate the application of the developed procedure using Daniel's data. The effects have been estimated in the usual manner and the results are displayed on the first column of Table III. The normalized IMAD is equal to 0.02 , so those effects whose estimates in absolute value exceed $0.088\left(4.4 M A D_{0}\right)$ will be considered active. According to this, effects 2, 4 and 8 are active.

The robust procedure and Box and Meyer's method have been applied to all four of the examples. Both methods found the same active effects in all cases. The active effects are also the same as those obtained when applying Daniel's method (Box and Meyer, 1986).

Figure 5 graphically illustrates the results for the four examples employing the robust procedure proposed in this article, indicating the intervals where the non-active effects are situated. The numbers correspond to the columns of the design.

In examples 1, 2 and 3 a dot plot of the estimated effects is sufficient to find which of the estimated effects are significant. Figure 5 shows that 2, 4 and 8 in example $I$ as well as 14 and 15 in example II and 4, 12 and 13 in III, are noticeably separated from the rest. In this case, to apply Box and Meyer's method would be an unnecessary complication.

The analysis of example IV (a full factorial design) involves more diffi- 
TABLE II. Four examples of 16-Run Two-Level factorial designs (I. Daniel (1976), II. Taguchi and Wu (1980), III. Box, Hunter and Hunter (1978), and IV. Davies (1954)).

\begin{tabular}{cccccccccccccccccccccccc} 
Run & 0 & 1 & 2 & 3 & 4 & 5 & 6 & 7 & 8 & 9 & 10 & 11 & 12 & 13 & 14 & 15 & I & II & III & IV \\
\hline 1 & + & - & - & + & - & + & + & - & - & + & + & - & + & - & - & + & 0.23 & 43.7 & 14.0 & 0.08 \\
2 & + & + & - & - & - & - & + & + & - & - & + & + & + & + & - & - & 0.30 & 40.2 & 16.8 & 0.04 \\
3 & + & - & + & - & - & + & - & + & - & + & - & + & + & - & + & - & 0.52 & 42.4 & 15.0 & 0.53 \\
4 & + & + & + & + & - & - & - & - & - & - & - & - & + & + & + & + & 0.54 & 44.7 & 15.4 & 0.43 \\
5 & + & - & - & + & + & - & - & + & - & + & + & - & - & + & + & - & 0.70 & 42.4 & 27.6 & 0.31 \\
6 & + & + & - & - & + & + & - & - & - & - & + & + & - & - & + & + & 0.76 & 45.9 & 24.0 & 0.09 \\
7 & + & - & + & - & + & - & + & - & - & + & - & + & - & + & - & + & 1.00 & 42.2 & 27.4 & 0.12 \\
8 & + & + & + & + & + & + & + & + & - & - & - & - & - & - & - & - & 0.96 & 40.6 & 22.6 & 0.36 \\
9 & + & - & - & + & - & + & + & - & + & - & - & + & - & + & + & - & 0.32 & 42.4 & 22.3 & 0.79 \\
10 & + & + & - & - & - & - & + & + & + & + & - & - & - & - & + & + & 0.39 & 45.5 & 17.1 & 0.68 \\
11 & + & - & + & - & - & + & - & + & + & - & + & - & - & + & - & + & 0.61 & 43.6 & 21.5 & 0.73 \\
12 & + & + & + & + & - & - & - & - & + & + & + & + & - & - & - & - & 0.66 & 40.6 & 17.5 & 0.08 \\
13 & + & - & - & + & + & - & - & + & + & - & - & + & + & - & - & + & 0.89 & 44.0 & 15.9 & 0.77 \\
14 & + & + & - & - & + & + & - & - & + & + & - & - & + & + & - & - & 0.97 & 40.2 & 21.9 & 0.38 \\
15 & + & - & + & - & + & - & + & - & + & - & + & - & + & - & + & - & 1.07 & 42.5 & 16.7 & 0.49 \\
16 & + & + & + & + & + & + & + & + & + & + & + & + & + & + & + & + & 1.21 & 46.5 & 20.3 & 0.23 \\
\hline
\end{tabular}

TABLE III. Estimated Effects in the four examples of Table II.

\begin{tabular}{|c|r|r|r|r|}
\hline Column & \multicolumn{1}{|c|}{ I } & II & III & IV \\
\hline 1 & .06 & .13 & -.6 & -.19 \\
2 & .25 & -.15 & -.4 & -.02 \\
3 & -.01 & .30 & -.6 & .00 \\
4 & .50 & .15 & 4.6 & -.08 \\
5 & .00 & .40 & .9 & .03 \\
6 & -.02 & -.03 & -.2 & -.07 \\
7 & .00 & .37 & -.3 & .15 \\
8 & .14 & .4 & -1.2 & .27 \\
9 & .03 & -.05 & .7 & -.16 \\
10 & -.01 & .42 & .1 & -.25 \\
11 & .02 & .13 & .3 & -.10 \\
12 & .04 & .13 & -5.5 & -.03 \\
13 & .02 & -.37 & 3.8 & -.01 \\
14 & .01 & 2.15 & .1 & .12 \\
15 & .02 & 3.10 & -.6 & .02 \\
\hline
\end{tabular}


culties. In the original publication (Davies, 1954), the three- and four-factor interactions are combined to give an estimate of error variance and, after carrying out a doubful analysis of variance, the authors assert that effects 8 and 10 are active. The normal probability paper method does not lead to a clear and evident solution; out of other possible interpretations the most reasonable is to consider that none of the effects are significant. Calculating a robust estimate of the variance of the estimates we reach the conclusion that none of the effects are active. Box and Meyer's method offers different results depending on the initial hypothesis, although considering the graph the authors decide that there is not sufficient evidence to conclude the existence of significant effects.

\section{CONCLUSIONS}

Unreplicated factorial designs can be analysed using various procedures: (1) a subjective method suggested by Daniel based on the normal probability paper, (2) an objective method proposed by Box and Meyer which requires very complex calculations and (3) other methods based on a robust estimator of the estimates' scale parameter.

This article proposes a very simple method which leads to the same results as the previous methods. It can be used as an alternative and complement to Daniel's graph and the Bayesian method.

Simulation experiments show that it is only necessary to iterate when the expected number of active effects is large, (larger than 20\%). In these cases, the proposed method has clear advantages over the one described by Lenth(1989).

From the simulations we conclude that it is advantageous to use as a scale estimator of a contaminated sample the robust estimator rather than that obtained through the residual variance of the model which only takes into account the estimated active effects. 


\section{APPENDIX}

\section{Limit Value of $I M A D_{0}$ in the Normal Case}

We will prove in this Appendix that the iterative process described in 4.1 converges in the limit $(n \rightarrow \infty)$ to a positive number $\left(a_{w}\right.$, the factor used for normalization) when $w>2$. Supposing that no significant effects exist and that $n$ is large (in the limit equal to $\infty$ ), the estimates of the effects can be seen as a random variable $X, \mathrm{~N}\left(0, \sigma^{2}\right)$. For $\sigma=1, T=|X|$ has the distribution function $F_{T}(t)=2 \Phi(t)-1, t \geq 0$ and its median is $t_{0}=\Phi^{-1}(3 / 4)$. From the first iteration we obtain a variable $X_{1}$, with normal standard distribution, truncated between $\left(-w t_{0}, w t_{0}\right)$, where $w=z_{c} / \Phi^{-1}(3 / 4)$, in such a way that $T=\left|X_{1}\right|$ has the following distribution function

$$
F_{T}(t)=\frac{2 \Phi(t)-1}{2 \Phi\left(w t_{0}\right)-1}, \quad 0 \leq t \leq w t_{0}
$$

and its median $t_{1}$ is obtained from the equation

$$
\Phi\left(t_{1}\right)=\frac{1}{2} \Phi\left(w t_{0}\right)+\frac{1}{4}
$$

which may be generalized for any iteration $m$

$$
\Phi\left(t_{m}\right)=\frac{1}{2} \Phi\left(w t_{m-1}\right)+\frac{1}{4}
$$

The succession $t_{m}$ generated is bounded and decreasing, so it converges. As $\Phi$ is continuous, the limit of the succession $t$ must verify equation (7). Such an equation has a trivial solution for $t=0$. Other solutions are the zeros of the function

$$
g(t)=\Phi(t)-\frac{1}{2} \Phi(w t)-\frac{1}{4}
$$

and as $g$ is continuous, $g(\infty)=1 / 4, g(0)=0$ and $g^{\prime}(0)=\frac{1}{\sqrt{2 \pi}}\left(1-\frac{w}{2}\right)$ then for $w>2$ the equation (7) has a non-trivial solution, $a>0$, satisfying $g(a)=0$. The form of $g^{\prime}(t)$ for $t>0$, allows us to conclude that the root is unique and positive, if $w>2$. For $1 \leq w \leq 2$ there is no solution apart from the trivial one; other values of $w$ are not reasonable. 
To conclude, it is necessary to prove that the succession $t_{m}$, when $w>2$, converges to the non-trivial solution $a>0$. It is sufficient to prove that $a$ is a lower bound of $t_{m}$. This will be demonstrated by induction. As $t_{0}=\Phi^{-1}(3 / 4)>a$, supposing that $t_{m}>a$, then $w t_{m}>w a$ and as $\Phi^{-1}$ is increasing, it verifies that

$$
\Phi^{-1}\left(\frac{\left.2 \Phi\left(w t_{m}\right)+1\right)}{4}\right)>\Phi^{-1}\left(\frac{2 \Phi(w a)+1)}{4}\right),
$$

where the left-hand side of the inequality is $t_{m+1}$ and the right-hand side is $a$, implying $t_{m+1}>a$, as we wished to prove.

\section{Limit Value of $I M A D_{0}$ in a Mixture of Normals}

If $X$ has a distribution $(1-\alpha) \mathrm{N}(0,1)+\alpha \mathrm{N}\left(0, k^{2}\right)$

truncated in $\left(-w t_{m-1}, w t_{m-1}\right)$ (as in the previous case, it is supposed $\sigma=1$ ), the distribution function of the random variable $T=|X|$ is

$$
F_{T}(t)=\frac{(1-\alpha)(2 \Phi(t)-1)+\alpha(2 \Phi(t / k)-1)}{(1-\alpha)\left(2 \Phi\left(w t_{m-1}\right)-1\right)+\alpha\left(2 \Phi\left(t_{m-1} / k\right)-1\right)} .
$$

the median $t_{n}$ is the value $F_{T}\left(t_{m}\right)=\frac{1}{2}$, so that

$$
\begin{aligned}
\Phi\left(t_{m}\right)= & \frac{1}{2(1-\alpha)}-\frac{\alpha}{1-\alpha} \Phi\left(\frac{t_{m}}{k}\right)+\frac{1}{4(1-\alpha)}\left((1-\alpha)\left(2 \Phi\left(w t_{m-1}\right)-1\right)\right. \\
& \left.+\alpha\left(2 \Phi\left(\frac{w t_{m-1}}{k}\right)-1\right)\right)
\end{aligned}
$$

and as $\Phi$ is continous, if $\left\{t_{m}\right\} \rightarrow t$, this limit must verify (8). Analogously to the normal case, it can be shown that $t_{m}$ converges to a solution $t \neq 0$ of the previous equation, when $w>2$.

\section{ACKNOWLEDGMENTS}

This research was supported by the Grant PB87-0808, DGICYT, Minis terio de Educación y Ciencia, Spain. We are grateful to the referees and associate editor for making many helpful comments and suggestions. 


\section{BIBLIOGRAPHY}

Benski, H.C.(1989). "Use of a Normality Test to Indentify Significant Effects in Factorial Designs," Journal of Quality Technology, 21, pp.174-178.

Box, G.E.P., Hunter, W.G. and Hunter, J.S (1978). Statistics for Experimenters. New York: John Wiley.

Box, G.E.P. and Meyer, R. (1986). "An Analysis for Unreplicated Fractional Factorials," Technometrics, 109, 11-18.

Daniel, C. (1959). "Use of Half-Normal Plots in Interpreting Factorial Two Level Experiments," Technometrics, 1, 311-341.

Daniel, C. (1976). Applications of Statistics to Industrial Experimentation. New York: John Wiley.

Davies, O.L. ed. (1954). The Design and Analysis of Industrial Experiments. London: Oliver and Boyd.

Hampel, F.R., Ronchetti, E.M., Rousseeuw, P.J. and Stahel, W.A. (1986). Robust Statistics: The Approach Based on Influence Functions. New York: John Wiley.

Iglewicz, B. (1983). "Robust Scale Estimators and Confidence Intervals for Location," In: Understanding Robust and Exploratory Data Analysis, D.C. Hoaglin, F. Mosteller and J.W. Tukey (eds.). New York: John Wiley.

Lenth, R.V. (1989). "Quick and Easy Analysis of Unreplicated Fatorials," Technometrics, 31, 469-473.

Olsson, D.M. (1979). "A Small Sample Test for Non-Normality," Journal of Quality Technology, 11, pp. 95-99.

Olsson, D.M. (1981). "Corrigenda," Journal of Quality Technology, 13, pp. 282. 
Peña, D. (1987). Estadística: Modelos y Métodos 2. Madrid: Alianza Universidad Textos.

Peña, D. and Juan, J. (1989). “Identifying Significant Effects in Unreplicated Fractional Factorial Designs," Bulletin of Inter. Statist. Institute, 47th Session, Vol. 2, 190-191.

Taguchi, G. and Wu, Y. (1980). Introduction to Off-line Quality Control, Central Japan Quality Control Association. Nagoya, Japan.

Wang, W. and Lawson, J. (1988). "Estimating the Error of an Effect in Unreplicated 2-Level Fractional Factorial Designs," Quality and Reliability Engineering International, 4, pp. 189-192. 

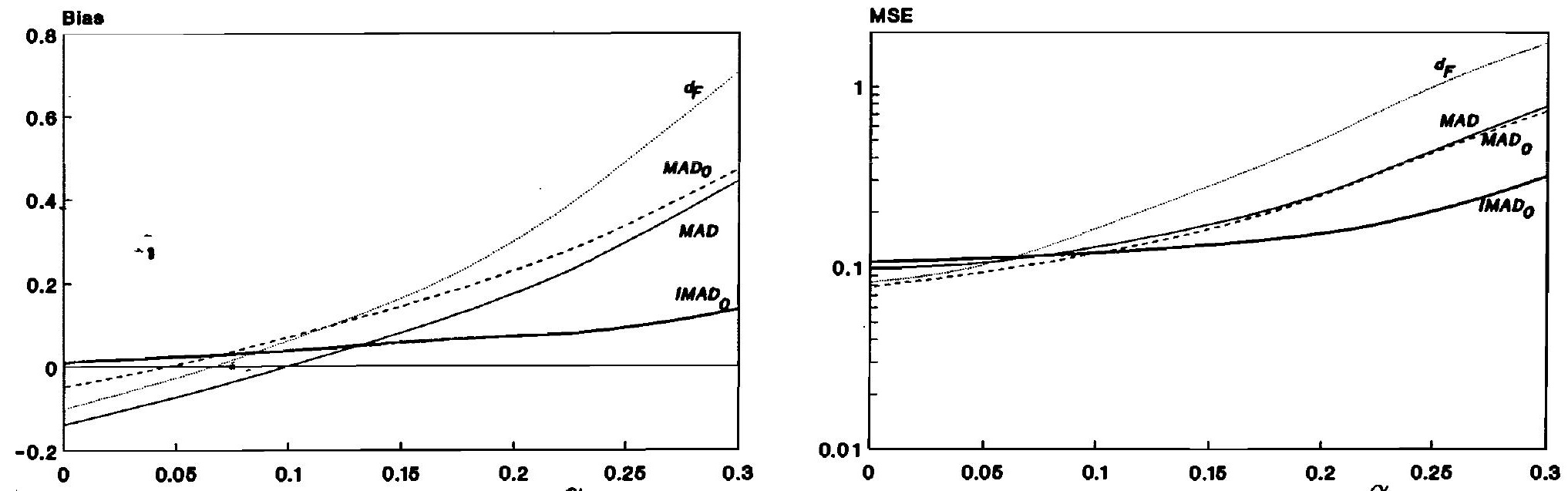

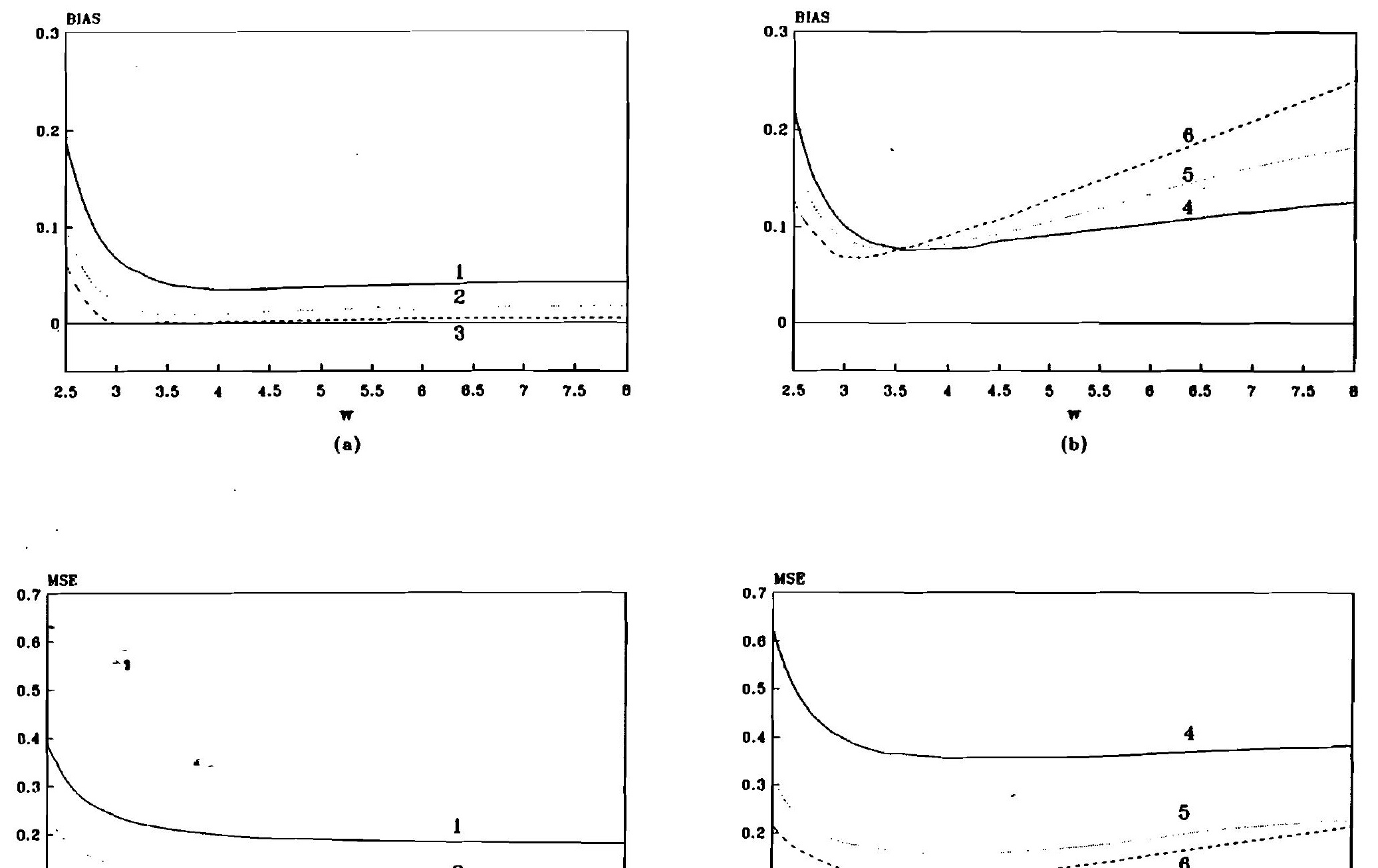


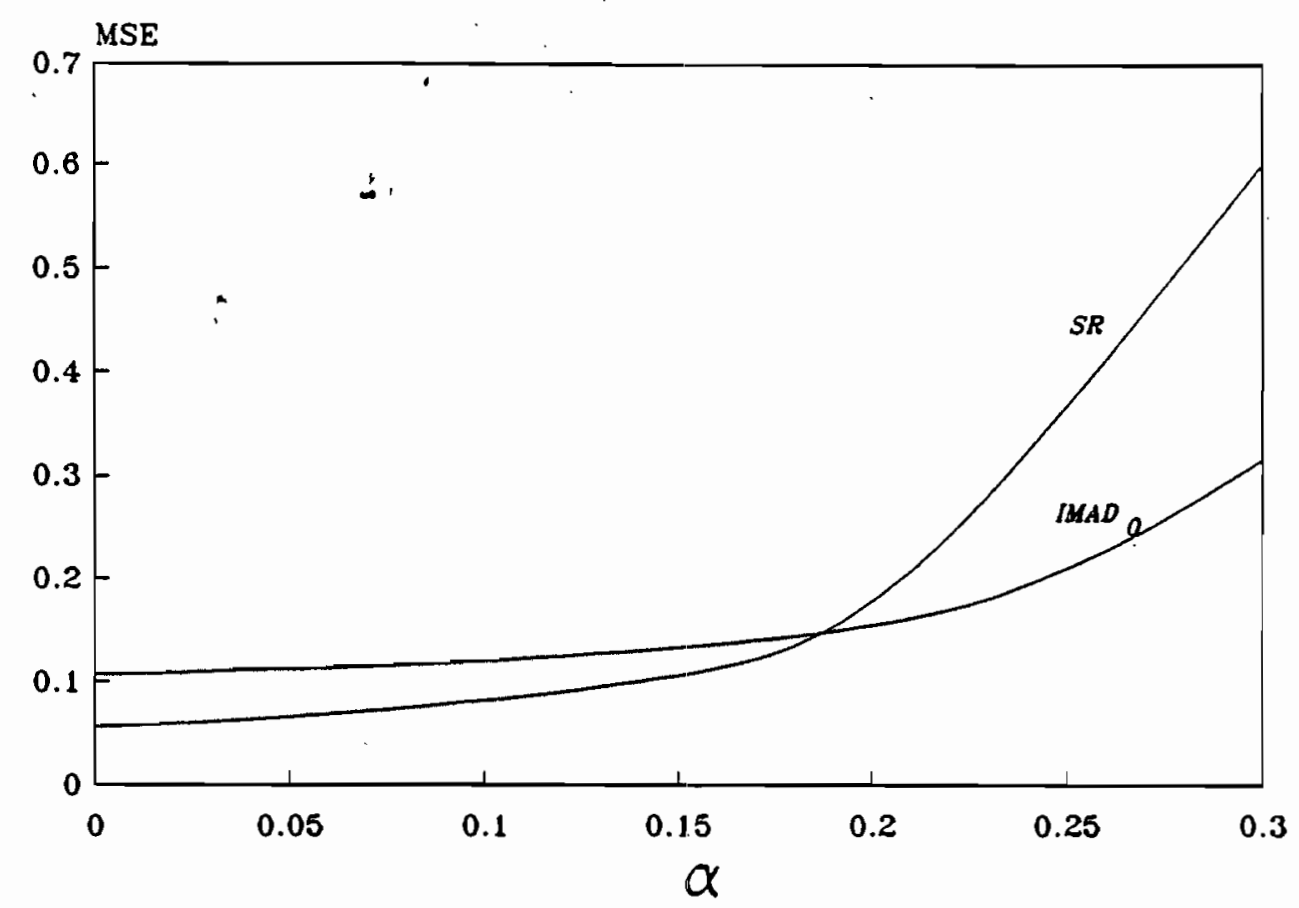

FIG. 3. Mean square error as a function of $\alpha$ for $s_{R}$ and $I M A D_{0}$ using 1000 samples of size 15 from $(1-\alpha) N(0,1)+\alpha N(0,100)$. 

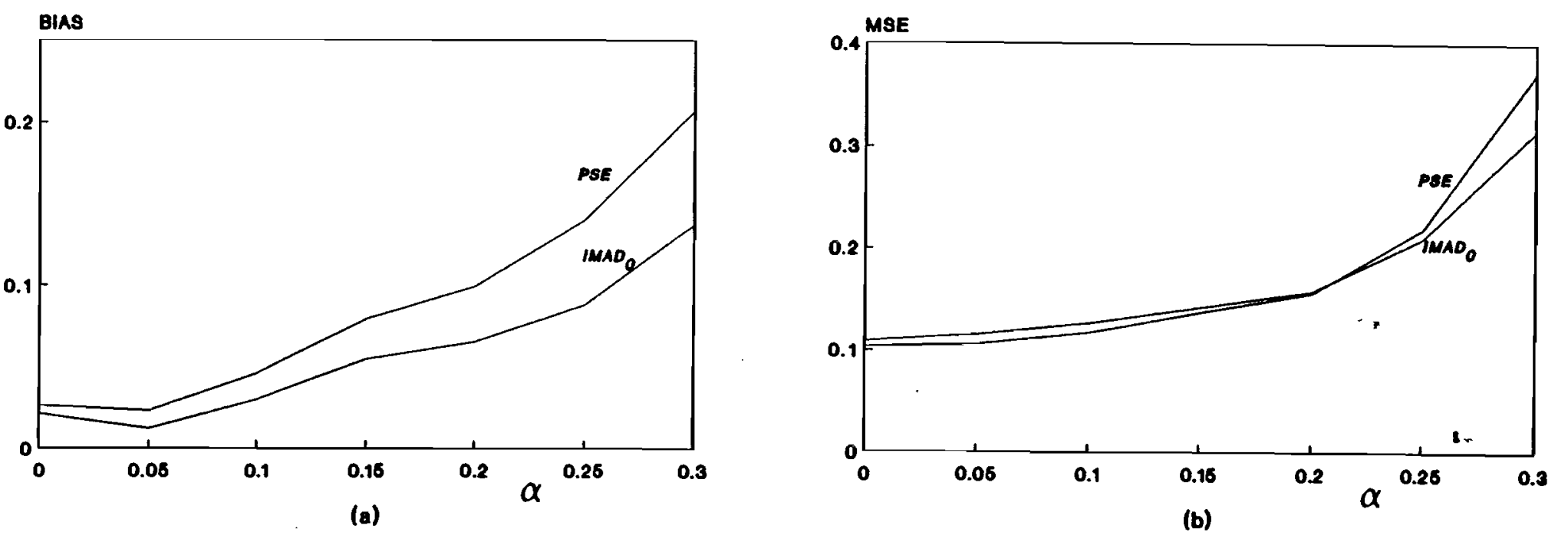

FIG. 4. (a) Estimated average bias as a function of $\alpha$ for $I M A D_{0}$ and $P S E$ using 1000 samples of size 15 from $(1-\alpha) N(0,1)+\alpha N(0,100)$. (b) Mean square error (MSE). 
I. Daniel (1976) $2^{4}$

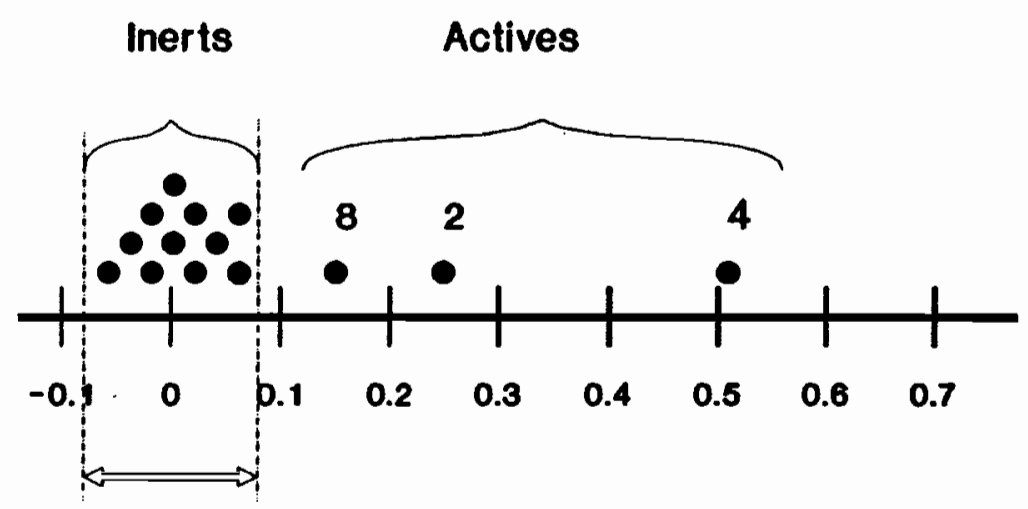

$(-0.089,0.089)$

III. Box, Hunter and Hunter (1978) $2_{\text {IV }}^{8-4}$

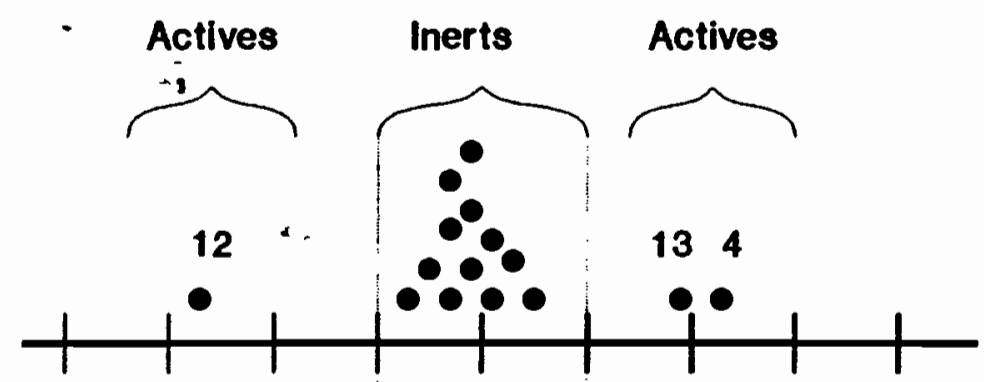

II. Taguchi and Wu (1980) $2_{\text {III }}^{9-6}$

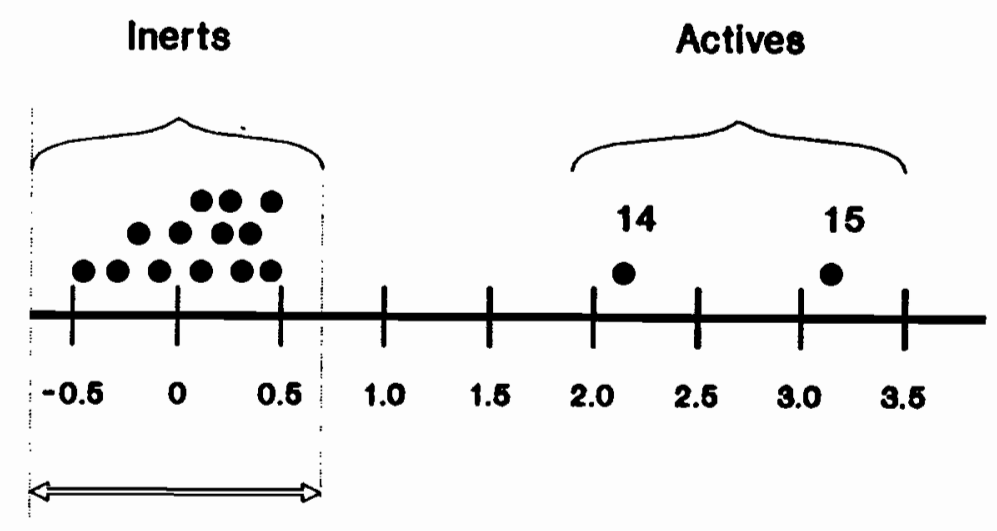

$(-0.67,0.67)$

IV. Davies (1954) $2^{4}$

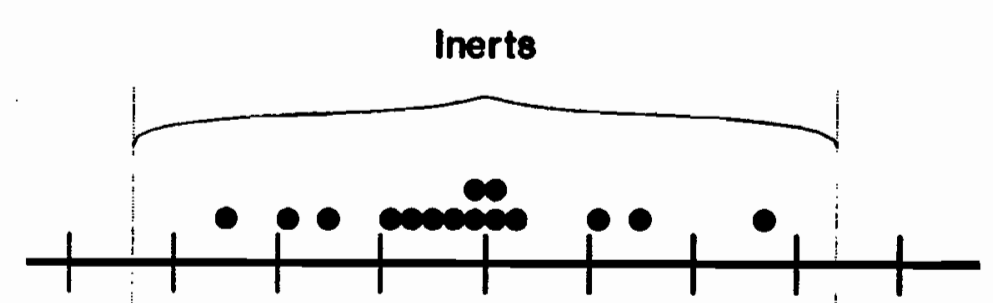

